\title{
«Schutz bei Hitzewelle» informiert über einfache Schutzmassnahmen
}

\author{
Betagte, Pflegebedürftige und Kleinkinder benötigen an Hitzetagen spezielle \\ Betreuung. Die Initiative «Schutz bei Hitzewelle» der Bundesämter für Gesund- \\ heit und Umwelt informiert gefährdete Personen und das Gesundheits- \\ personal.
}

Rita Molla

Andreas Biedermann ${ }^{b}$,

Thomas Pfluger

a Ärztinnen und Ärzte für Umweltschutz, Geschäftsleiterin

b Ärztinnen und Ärzte für Umweltschutz, Mitglied der Geschäftsleitung

c Projektleiter «Schutz bei Hitzewelle»
Korrespondenz:

Andreas Biedermann

Public Health Services

Güterstrasse 12

CH-3360 Herzogenbuchsee

Tel. 0629562323

biedermann@

public-health-services.ch
Der Hitzesommer 2003 hat auch in der Schweiz Todesopfer gefordert [1]. Nach Ansicht der meisten Klimaforschenden wird der Treibhauseffekt zu vermehrten Hitzewellen wie jener im Sommer 2003 führen. Studien haben gezeigt, dass dauernde Hitze eine Gefahr für die Gesundheit darstellt, besonders für betagte Personen und Menschen mit Lungen- und Herz-KreislaufKrankheiten, aber auch für Kleinkinder $[1,2]$. Das Bundesamt für Gesundheit (BAG) und das Bundesamt für Umwelt (BAFU) geben deshalb unter dem Motto «Schutz bei Hitzewelle» Informationsmaterial heraus, um Angehörige, Pflegepersonal, Ärzteschaft und gefährdete Personen für die gesundheitlichen Risiken der Hitze und die entsprechende Vorsorge $\mathrm{zu}$ sensibilisieren. Dabei werden sie von mehreren Kantonen und über 20 Partnerorganisationen unterstützt, unter anderem von der FMH und den Ärztinnen und Ärzten für Umweltschutz. Die im Rahmen der Kampagne abgegebenen Flugblätter und Poster orientieren praxisbezogen über wichtige Vorsorgemassnahmen (siehe Kasten), listen die Symptome von Hitzefolgen auf, geben Handlungsanweisungen und liefern Hintergrundinformationen.

\section{Hitze und Ozon}

Säuglinge und Kleinkinder können sich weniger gut an hohe Temperaturen anpassen als Erwachsene. Die existierenden Untersuchungen zeigen jedoch kein einheitliches Bild [2]. Laut einer südafrikanischen Studie war in der heissen Saison die Sterblichkeit bei Kleinkindern unter einem Jahr und bei Betagten erhöht [3], laut anderen nur bei Betagten [2]. Während der Hitzewelle 2003 gab es in Frankreich weder vermehrt pädiatrische Todesfälle noch mehr Spitaleinweisungen, die frei praktizierenden AllgemeinmedizinerInnen behandelten aber mehr Säuglinge und Kleinkinder [4]. Kleinkinder sind gemäss einer amerikanischen Untersuchung bei Hitzewellen gefährdet, insbesondere wenn sie an Durchfall, Infektionen der Atemwege oder neurologischen Erkrankungen leiden [5]. Für die Schweiz gibt es noch keine entsprechenden Untersuchungen.

Eine Studie aus Belgien hat ergeben, dass sich die negativen Auswirkungen von Hitze und Ozon gegenseitig verstärken [6]. Der Luftschadstoff reizt die Schleimhäute, kann Entzündungen der Atemwege und Lungen auslösen, die Lungenfunktion vorübergehend einschränken, die körperliche Leistungsfähigkeit beeinträch-

\footnotetext{
Die wichtigsten Verhaltensregeln bei Hitzewellen

- Körperliche Anstrengungen vermeiden

- Wohnung und Körper möglichst kühl halten

- Viel trinken (auf ausreichende Salzzufuhr achten)

- Erfrischende Speisen zu sich nehmen

Zusätzliche Regeln für Kleinkinder

- Vor Sonnenstrahlung schützen (Schatten, Kleider, Hut, Sonnencreme)

- Hauttemperatur regelmässig prüfen

- Spezieller Augenmerk auf Kleinkinder mit Durchfall

- Spezieller Augenmerk auf Kleinkinder mit Asthma und anderen chronischen Krankheiten

- In Wäldern und Parks mit dichtem Baumbestand spielen lassen

- Kinder nie unbeaufsichtigt in Autos oder in verschlossenen Räumen lassen
} 
tigen und Atembeschwerden auslösen. Zudem verstärkt er die Reaktion auf andere Luftschadstoffe und Allergene. Besonders gefährdet sind Kinder mit Atemwegskrankheiten; ein bestehendes Asthma kann verstärkt werden.

Das Material von «Schutz bei Hitzewelle» umfasst je ein Merkblatt für das Gesundheitspersonal und Angehörige bzw. für gefährdete Personen, einen Poster mit den wichtigsten Verhaltensregeln und drei Factsheets mit Hintergrundinformationen zum Auflegen, Aufhängen, Verteilen. Bestelladresse: Sektion Gesundheit und Umwelt, Bundesamt für Gesundheit BAG, 3003 Bern.

Weitere Informationen: www.hitzewelle.ch.

\section{Literatur}

1 Thommen O, et al. Mögliche gesundheitliche Auswirkungen des Klimawandels in der Schweiz. Schweiz Ärztezeitung 2005;86(21):1281-5.

2 Basu R, Samet JM. Relation between elevated ambient temperature and mortality: a review of the epidemiologic evidence. Epidemiol Rev 2002;24:190-202.

3 Wyndham CA, Fellingham SA. Climate and disease. S Afr Med J 1978;53:1051.

4 Pascal L, et al. Evaluation de l'impact de la vague de chaleur de l'été 2003 sur la morbidité hospitalière infantile. Institut de veille sanitaire (France), 2005.

5 Jones TS, et al. Morbidity and mortality associated with the July 1980 heat wave in St Louis and Kansas City, Mo. JAMA 1982;247:3327.

6 Sartor F, et al. Temperature, ambient ozone levels, and mortality during summer 1994, in Belgium. Environ Res 1995;70:105. 